\title{
Le Paléolithique inférieur et moyen en Béarn oriental
}

\section{Françoise Millet et Dominique Millet}

\section{OpenEdition \\ Journals}

Édition électronique

URL : http://journals.openedition.org/adlfi/7731

ISSN : 2114-0502

Éditeur

Ministère de la culture

Référence électronique

Françoise Millet et Dominique Millet, « Le Paléolithique inférieur et moyen en Béarn oriental », ADLFI. Archéologie de la France - Informations [En ligne], Aquitaine, mis en ligne le 01 mars 2007, consulté le 20 avril 2019. URL : http://journals.openedition.org/adlfi/7731

Ce document a été généré automatiquement le 20 avril 2019

(C) Ministère de la Culture et de la Communication, CNRS 


\title{
Le Paléolithique inférieur et moyen en Béarn oriental
}

\author{
Françoise Millet et Dominique Millet
}

Identifiant de l'opération archéologique : 025107

Date de l'opération : 2007 (PT)

1 La seconde tranche du programme concernait la zone sud est et nord cône de Ger sur les cantons de Lembeye, Pontacq, Montardon et Garlin et a concerné 33 communes. Un premier sondage d'évaluation a été pratiqué sur la commune de Moncaup (canton de Lembeye).

\section{Résultats obtenus}

\section{Données anthropogéographiques}

3 Le maillage des implantations fait ressortir des secteurs attractifs principalement localisés sur la bordure méridionale du cône de Ger, et la nappe de Limendous (bassin inférieur de la Souye, vallée du Gabas et du Luy-de-France) dans son extension septentrionale. La zone centrale centrée sur le complexe hydrographique du Lées semble en revanche constituer un domaine plutôt répulsif ou défavorable pour des raisons encore inconnues.

\section{Séquences chronostratigraphiques}

5 Les caractéristiques taphonomiques (épandage, écrêtement, travaux du Génie rural, compilation naturelle possible d'horizons stratigraphiques dans les séquences reconnues) comparables qui affectent l'ensemble des implantations ne permettent pas d'affirmer que les assemblages recueillis soient exempts de mélanges. Un sondage d'évaluation a eu lieu à Moncaup (64) au lieu-dit «Lasserre ». La séquence lithologique suivante contenait un horizon avec du matériel archéologique (voir fig.) : 

Les unités 1 et 2 correspondent à différents niveaux pléistocènes, l'unité 3 au tertiaire. Du matériel archéologique (outillage sur éclat, galet taillé, produits de débitage, nucléus) proviennent de la surface du cailloutis de l'unité 1 . Certaines pièces très altérées ont subi un épandage ; d'autres, seulement patinées, proviennent de la masse du cailloutis cimenté par un « grep » résistant. Ailleurs (secteur méridional et occidental de la zone prospectée) la séquence suivante a été reconnue (ép. : 1,6 m au maximum) :

160 - Horizon agricole remanié ou parfois Terre à Touyas ;

172 - Niveaux limono-argileux jaune-brun pédogénisés enrichis progressivement en argile et granules ferromanganique (Fe-Mn) vers la base ;

183 - Sommet de la nappe de Limendous rubéfié et localement cimenté par des dépôts FeMn caractéristiques d'anciennes zones de battement;

194 - Sommet de la nappe de Limendous proprement dit.

20 Le matériel archéologique porte le plus souvent des altérations pédologiques correspondant à celles rencontrées dans les couches 2 et 3.

21 Caractérisation chronoculturelle des occupations en trois composantes

22 Compte tenu de l'absence de références locales, nous avons adopté les critères typotechnologiques associés à la nature et l'intensité des stigmates d'altération comme caractéristiques discriminantes.

23 Les composantes chronoculturelles identifiées confirment les résultats obtenus dans la première tranche :

24 - Acheuléen plein (voir fig. 1) caractérisé par un outillage bifacial (bifaces, hachereaux, pièces à section triédrique) façonné sur de gros éclats d'entames surdéterminés. Cet outillage lourd est souvent associé à des galets taillés (choppers surtout distaux). Le débitage recueilli comporte surtout de gros supports, épais obtenus soit par fendage de galet, soit débitage unidirectionnel prismatique sur enclume. Les surfaces de ces pièces sont très altérées (rubéfaction, dissolution, encroûtement ferromanganique, etc.). Certaines sont parfois roulées incorporées à des terrasses du Pléistocène moyen.

- Acheuléen évolué déjà engagé sur des processus technoéconomiques de type Paléolithique moyen. L'outillage bifacial demeure bien représenté, exploitant aussi bien les galets que les supports surdéterminés. La percussion lourde (percuteurs mobiles et enclumes) est associée à l'utilisation de la percussion tendre pour la finition des pièces Les modules sont inférieurs à ceux de la série précédente. L'outillage lourd sur éclat est dominé par les racloirs sur face plane réalisés sur de gros éclats d'entame corticaux. Les chaînes opératoires de débitage s'allongent (récurrence) et s'articulent principalement 
autour de la variabilité du concept discoïde. Quelques nucléus Levallois à débitage préférentiel figurent dans les séries. La production de supports mieux calibrés (épaisseur du talon surtout naturel) émerge. Aucune pièce roulée ne figure dans les assemblages de cette série.

- Paléolithique moyen est assez indéterminé. Il présente des affinités avec une forme de Moustérien de tradition Acheuléenne dans lequel les hache-hachereaux très stéréotypés sont présents au détriment des bifaces (faciès régional vasconien si ce dernier existe vraiment). L'obtention de supports en est la principale caractéristique. Ce matériel est assez «frais ", faiblement altéré par la pédogénèse. Les chaînes opératoires de débitage sont en général assez courtes et peu ramifiées. Les variabilités discoïdes et unipolaires prismatiques dominent.

(Fig. $\mathrm{n}^{\circ} 1$ : Lasclaveries - Hachereau de gros module sur éclat surdéterminéMoncaup - Séquence lithologique du sondage 2007 (pf. 2,4 m))

\section{ANNEXES} du Lées) ;

\section{Objectifs pour la campagne 2008}

Cette dernière tranche s'articulera selon trois axes majeurs :

- l'évaluation des potentialités reconnues pour les implantations présentant encore des garanties stratigraphiques (série de sondages à Moncaup dans la vallée du Louet) ;

- vérification de l'extension de l'occupation dans la partie septentrionale et occidentale de la zone d'étude (cantons de Thèze, Garlin avec les vallées du Luy de France, du Gabas et

- recherche et inventaire de séquences stratigraphiques.

Millet Dominique et Françoise 
Fig. $\mathrm{n}^{\circ} 1$ : Lasclaveries - Hachereau de gros module sur éclat surdéterminéMoncaup - Séquence lithologique du sondage 2007 (pf. 2,4 m)

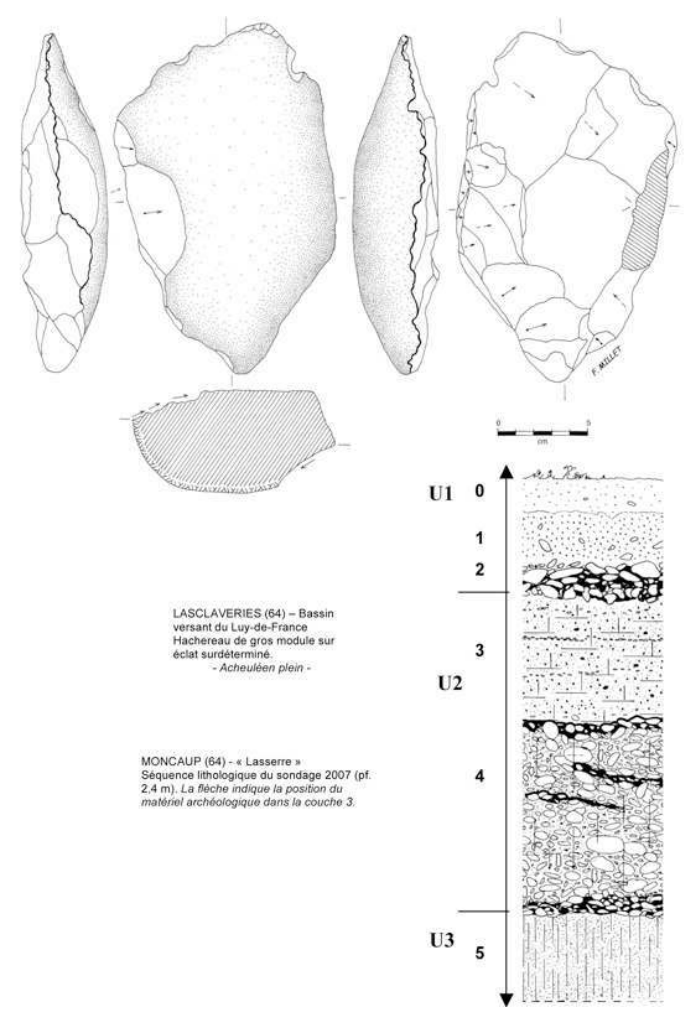

Auteur(s) : Millet, Dominique (SUP). Crédits : Millet Dominique (2007)

INDEX

Index géographique : Aquitaine, Pyrénées-Atlantiques (64), Lembeye, Pontacq, Montardon, Garlin

operation Prospection thématique (PRT)

Thèmes : anthropologie de terrain, chaîne opératoire, chronostratigraphie, débitage, éclat, galet, hache, hachereau, industrie lithique, nucleus, outil, technique Levallois

Index chronologique : Paléolithique inférieur, Paléolithique moyen

\section{AUTEURS}

FRANÇOISE MILLET

BEN

DOMINIQUE MILLET

SUP 